\title{
INTERGROWTH-21st v. local South African growth standards (Theron-Thompson) for identification of small-for-gestational-age fetuses in stillbirths: A closer look at variation across pregnancy
}

\author{
T Lavin, ${ }^{1} \mathrm{MPH}$, MIntHlth, BSc Hons; L Nedkoff, ${ }^{2} \mathrm{PhD}, \mathrm{MPH}$, GradDipBus, BAppSc; D Preen, ${ }^{1} \mathrm{PhD}$, BSc Hons; \\ G Theron, ${ }^{3}$ MB ChB, FCOG (SA), MMed (O\&G), Hons BSc (Epidemiol), MD; R C Pattinson, ${ }^{4}$ MD, FRCOG, FCOG (SA
${ }^{1}$ Centre for Health Services Research, School of Population and Global Health, Faculty of Health and Medical Sciences, University of Western Australia, Australia
${ }^{2}$ Cardiovascular Research Group, School of Population and Global Health, Faculty of Health and Medical Sciences, University of Western Australia, Australia
${ }^{3}$ Department of Obstetrics and Gynaecology, Faculty of Medicine and Health Sciences, Stellenbosch University, Cape Town, South Africa ${ }^{4}$ South African Medical Research Council Maternal and Infant Health Care Strategies Unit, Department of Obstetrics and Gynaecology, School of Medicine, Faculty of Health Sciences, University of Pretoria, South Africa

Corresponding author: T Lavin (tina.lavin@uwa.edu.au)

\begin{abstract}
Background. Global growth standards for fetuses were recently developed (INTERGROWTH-21st). It has been advocated that professional bodies should adopt these global standards.

Objectives. To compare the ability of INTERGROWTH-21st with local standards (Theron-Thompson) to identify small-for-gestational-age (SGA) fetuses in stillbirths in the South African (SA) setting.

Methods. Stillbirths across SA were investigated (>500 g, 28 - 40 weeks) between October 2013 and December 2016 ( $N=14$ 776). The study applied the INTERGROWTH-21st standards to classify stillbirths as <10th centile (SGA) compared with Theron-Thompson growth charts, across pregnancy overall and at specific gestational ages.

Results. The prevalence of SGA was estimated at $32.2 \%$ and $31.1 \%$ by INTERGROWTH-21st and Theron-Thompson, respectively. INTERGROWTH-21st captured $13.8 \%$ more stillbirths as SGA in the earlier gestations ( 28 - 30 weeks, $p<0.001)$, but $4.0 \%(n=315)$ fewer between 33 and 38 weeks $(p<0.001)$. Observed agreement and the Kappa coefficient were lower at earlier gestations and at $34-36$ weeks. Conclusions. Our findings demonstrated differences in the proportion of stillbirths considered SGA at each gestational age between the INTERGROWTH-21st and the local SA standard, which have not been considered previously by other studies.

S Afr Med J 2019;109(7):519-525. DOI:10.7196/SAMJ.2019.v109i7.13485
\end{abstract}

Fetuses that are small for gestational age (SGA) are at increased risk of stillbirth. SGA fetuses that are not detected during the antepartum period are at a four-fold increased risk of serious fetal complications compared with SGA fetuses detected before delivery. ${ }^{[1]}$ It is therefore important to identify SGA pregnancies antenatally so that complications and stillbirth risk can be reduced through appropriate monitoring and clinical care.

Traditionally, country-specific population fetal growth charts have been used to identify SGA infants. However, there is substantial intercountry variation between growth charts, meaning that a fetus whose growth is tracking as appropriate using one particular chart may be classified as growth restricted under another. ${ }^{[2]}$ Recently a global, multiethnic standardised chart for fetal growth and size was developed by the International Fetal and Newborn Growth Consortium for the 21st Century (INTERGROWTH-21st). ${ }^{[3,4]}$ INTERGROWTH-21st is intended for global use and improves comparison between countries. ${ }^{\left[{ }^{[}\right]}$ It has been advocated that this new standard should be adopted by professional bodies ${ }^{[5]}$ but uptake has been variable to date. The ability of INTERGROWTH-21st to identify SGA in stillbirth cases compared with local South African (SA) standards (Theron-Thompson growth charts) has not been assessed.
Studies comparing local growth standards with INTERGROWTH21st have mainly been conducted in high-resource countries such as New Zealand (NZ) and the UK. ${ }^{[6,7]}$ These studies have shown that INTERGROWTH-21st underestimated SGA in at-risk infants in $\mathrm{NZ}^{[6]}$ and stillbirths in the UK ${ }^{[7]}$ compared with local standards. The NZ study showed that INTERGROWTH-21st was specifically less able to detect at-risk SGA infants compared with local customised standards among some ethnic groups, including Maori, European and Pacific women ${ }^{[6]}$ In a middle-income setting, a Chinese study found that INTERGROWTH-21st overestimated the proportion of SGA liveborn infants compared with local standards, resulting in an increase in the number of pregnancies requiring further investigations to ascertain fetal wellbeing. ${ }^{[8]}$

The variation observed in classifying at-risk SGA infants using INTERGROWTH-21st growth standards, in particular for different ethnicities ${ }^{[6]}$ suggests that investigation to compare INTERGROWTH-21st with local Theron-Thompson standards in the SA population of stillbirths is warranted. Further, there is limited published literature on the impact of gestational age on the agreement of INTERGROWTH-21st and local fetal growth charts in identifying SGA infants. 


\section{Objectives}

The primary objective of this study was to determine the proportion of stillbirths classified as SGA using INTERGROWTH-21st compared with local Theron-Thompson growth charts. The study allowed for comparison of the proportion of stillbirths classified as SGA in the SA population with global estimates for the first time. The secondary objective was to determine whether there were differences in the proportion of stillbirths identified as SGA by gestational age.

\section{Methods}

A secondary analysis of all stillbirths across SA ( $>500 \mathrm{~g}$ and $\geq 28$ weeks $\leq 40$ weeks) between October 2013 and December 2016 was conducted. The SA Perinatal Problems Identification Program (PPIP) database was used for this study, capturing $>90 \%$ of deaths across all health facility levels in SA. PPIP is a perinatal quality audit system that has been described in detail elsewhere. ${ }^{[9]}$ Briefly, after each perinatal death the clinical team performs a review and records clinical information around the cause of death as well as weight/ gestational age. Gestational age is determined based on the date of the last menstrual period (LMP), ultrasound or clinical examination. Stillbirths are classified as macerated, which are clinically diagnosed when the skin of the fetus is discoloured, blotchy and friable to touch, or as fresh when the skin is intact and 'normal' in appearance.

Data were extracted in aggregate form at health facility level. Stillbirth cases were excluded if the gestational age was unknown ( $n=20786,46.9 \%)$ or if the estimated age was considered 'uncertain' $(n=8750,19.7 \%)$.

\section{Theron-Thompson growth charts}

Theron-Thompson growth charts were developed in 1995 using an urban population in Western Cape Province, SA $\left(N=3\right.$ 643). ${ }^{[10]}$ It included women who presented antenatally at the Tygerberg Hospital obstetric service (including attached community clinics), representing half of all women who delivered in the circumscribed urban area. The mean age of the group was 25.1 years (range 14 46), $40.6 \%$ were primigravidas, and $92.1 \%$ were of coloured ethnicity, $4.3 \%$ white, $3.4 \%$ black and $0.2 \%$ Asian. Gestational age was confirmed by early ultrasound. Centile charts for birth weight by gestational age were constructed for this population.

\section{Classification as SGA}

PPIP automatically classifies cases below the 10th centile using Theron-Thompson growth charts as SGA. The classification of stillbirths as SGA using INTERGROWTH-21st was performed by applying the $<10$ th centile birth-weight cut-off at each gestational age.

\section{Statistical analysis}

The prevalence of SGA was calculated for the study sample as the number of SGA stillbirths divided by the total number of stillbirths and presented as a proportion. It was calculated for TheronThompson and INTERGROWTH-21st separately.

Observed agreement for identifying SGA infants using INTERGROWTH-21st and Theron-Thompson growth charts was calculated. The Kappa coefficient $(\kappa)$ and $95 \%$ confidence intervals were calculated for concordance between identification of SGA using INTERGROWTH-21st and Theron-Thompson. Observed agreement and $\kappa$ were calculated overall across pregnancy (28 40 weeks) and individually at each gestational age (weeks). This was done separately for all stillbirths, macerated stillbirths, fresh stillbirths and intrauterine growth-restricted (IUGR) stillbirths.

Pearson's $\chi^{2}$ test or Fisher's exact test $($ where $n<5)$ were used to compare the crude proportions of SGA stillbirths between TheronThompson and INTERGROWTH-21st at each gestational age (in weeks). Comparisons were made at each gestational age for all stillbirths, and separately for macerated and fresh stillbirths. In addition, stillbirths with a confirmed primary cause of death as IUGR were examined as a separate cohort. We also made comparisons between methods of gestational age determination (LMP, ultrasound or clinical examination), as well as separate analyses by method of gestational age determination. The proportion of SGA stillbirths at each gestational age compared between Theron-Thompson and INTERGROWTH-21st was tested using Pearson's $\chi^{2}$ test.

\section{Results}

There were 14776 eligible stillbirths (after exclusion for unknown gestational age) during the study period (9 725 macerated, 5051 fresh). There were no statistically significant differences between the included and excluded cases in terms of maternal age, parity, HIV status or syphilis status. There were 9389 stillbirths (63.5\%) not classified as SGA using any criteria. A total of $30.1 \%$ of stillbirths $(n=4452)$ were classified as SGA using both criteria, 3.2\% $(n=465)$ were identified by INTERGROWTH-21st only and $2.1 \%(n=315)$ were identified by Theron-Thompson only; $1.1 \%(n=155)$ were classified as appropriate for gestational age (AGA) by TheronThompson only.

\section{Sociodemographic characteristics}

Clinical characteristics of the study cohort are presented in Table 1. Compared with stillbirths that were classified as SGA (both criteria), mothers in the group classified as AGA (using both criteria) were healthier and more frequently primiparous and included fewer HIVpositive cases and fewer preterm births. The SGA INTERGROWTH-

Table 1. Clinical characteristics of SGA stillbirth cases by method of classification, SA, October 2013 - December 2016 ( $N=14776)$

\begin{tabular}{|c|c|c|c|c|c|c|}
\hline & $\begin{array}{l}\text { All stillbirths } \\
(N=14776), n(\%)\end{array}$ & $\begin{array}{l}\text { SGA INTERGROWTH- } \\
21 \text { st only }(N=465) \text {, } \\
n(\%)\end{array}$ & $\begin{array}{l}\text { SGA Theron- } \\
\text { Thompson only } \\
(N=325), n(\%)\end{array}$ & $\begin{array}{l}\text { Both SGA }^{\dagger} \\
(N=4 \text { 452), } n(\%)\end{array}$ & $\begin{array}{l}\operatorname{AGA}^{\ddagger}(N=9389), \\
n(\%)\end{array}$ & $\begin{array}{l}\text { AGA Theron- } \\
\text { Thompson only } \\
(N=155), n(\%)\end{array}$ \\
\hline 'Healthy' mother ${ }^{\S}$ & $8182(55.4)$ & $253(52.2)$ & $131(41.5)$ & $2355(52.9)$ & $5239(55.8)$ & $88(56.8)$ \\
\hline Primipara & $6076(41.1)$ & $199(42.8)$ & $125(38.3)$ & $1812(40.7)$ & $3877(41.3)$ & $81(52.3)$ \\
\hline Received antenatal care & $14096(95.4)$ & $436(93.7)^{\star}$ & $304(96.5)$ & $4234(95.1)$ & $8508(95.1)$ & $136(87.7)$ \\
\hline HIV (positive) & $4104(27.7)$ & $193(41.5)^{\star}$ & $86(27.3)$ & $1296(29.1)$ & 2525 (26.9) & $90(58.1)$ \\
\hline Preterm birth & $9530(64.5)$ & $428(92.0)^{*}$ & $230(73.0)$ & $3103(69.7)$ & $5689(60.5)$ & $95(61.3)$ \\
\hline
\end{tabular}


21st-only group included more healthy, primparous mothers, fewer mothers who had received antenatal care, more HIVpositive mothers and a higher proportion of preterm births (Table 1).

\section{Prevalence of SGA}

The prevalence of SGA was estimated at $32.2 \% \quad(n=4753)$ and $31.1 \% \quad(n=4598)$ by INTERGROWTH-21st and TheronThompson criteria, respectively ( $p=0.052)$. In IUGR fetuses $(n=384)$, the incidence of SGA was $78.1 \%(n=300)$ using INTERGROWTH21 st and $77.6 \%(n=298)$ using TheronThompson $(p=0.058)$.

\section{Observed agreement and $\kappa$}

Overall across pregnancy, observed agreement between the methods of SGA determination was high (98.9\%); $\kappa$ was also high at 0.976 . Similar results were seen for fresh $(99.3 \%, \kappa 0.982)$ and macerated (98.8\%, к 0.968) stillbirths (Table 2). When considering each gestational age separately, the lowest observed agreement was at lower gestations (28 and 29 weeks $76.4 \%$ and $86.3 \%$, respectively, and $34-36$ weeks $94.6 \%, 92.5 \%$ and $94.7 \%$, respectively). For stillbirths with IUGR as a primary cause of death, observed agreement was lower at 28 and 29 weeks $(67.7 \%, 93.3 \%)$ and 37 39 weeks $(95.5 \%, 94.1 \%, 86.7 \%)$ compared with other gestations. Overall agreement across pregnancy was high at $99.5 \%$ ( $\kappa$ 0.985) for IUGR deaths.

\section{Proportion of SGA stratified by gestational age}

A comparison of INTERGROWTH-21st and Theron-Thompson standards across gestation can be seen in Fig. 1. When considering each gestational age (weeks) separately, there were differences in the proportion of SGA fetuses classified as SGA between INTERGROWTH-21st and TheronThompson. INTERGROWTH-21st captured $13.8 \%(n=437)$ more stillbirths as SGA in the earlier gestations (28 - 30 weeks; $p<0.001$ ) but $4.0 \%(n=315)$ fewer at gestations between 33 and 38 weeks $(p<0.001)$ compared with Theron-Thompson (Fig. 2, A). This trend was observed for both macerated and fresh stillbirths (Fig. 2, B and C).

In pregnancies with IUGR as a primary cause of death, INTERGROWTH-21st classified $16.0 \%$ more stillbirths as SGA at younger gestations (28 - 30 weeks) and 9.4\% fewer at 38 - 39 weeks compared with Theron-Thompson (Fig. 3). However, the only statistically significant difference was at 28 weeks $(p=0.011)$.

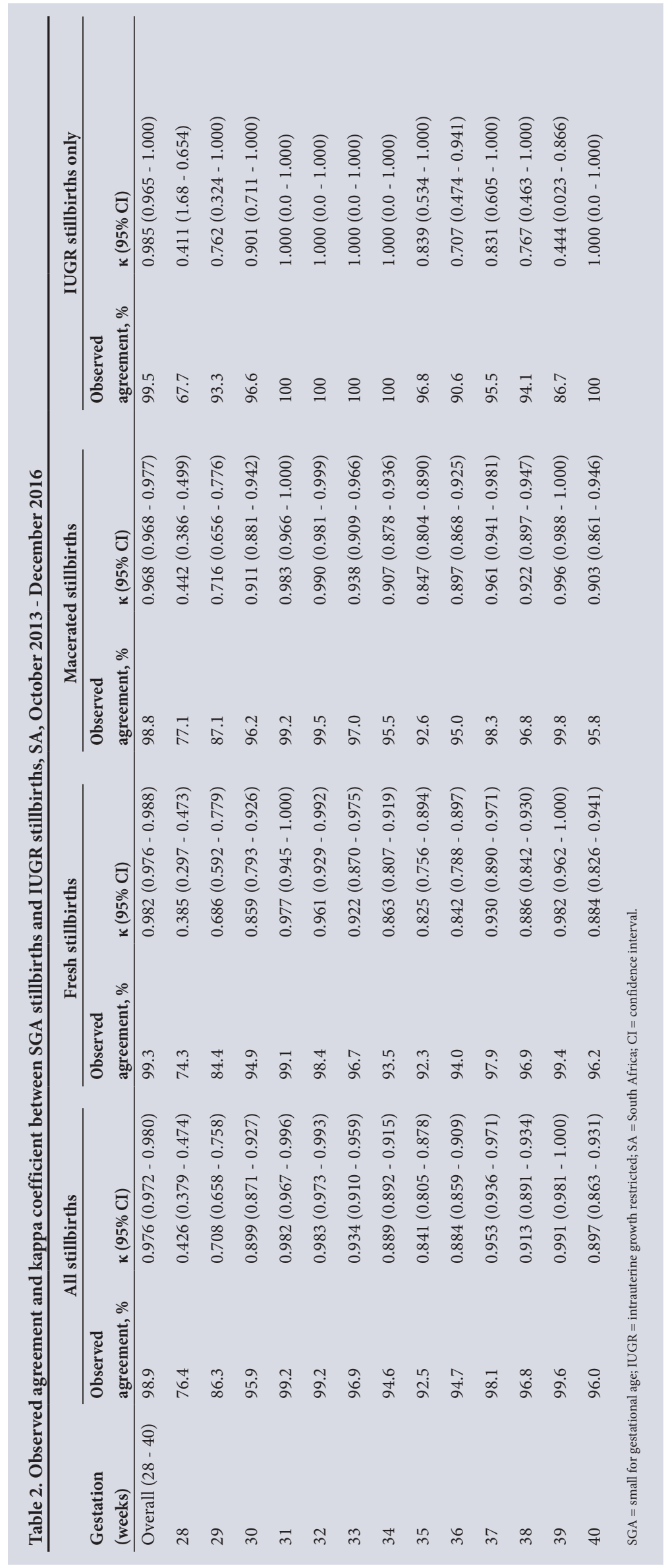




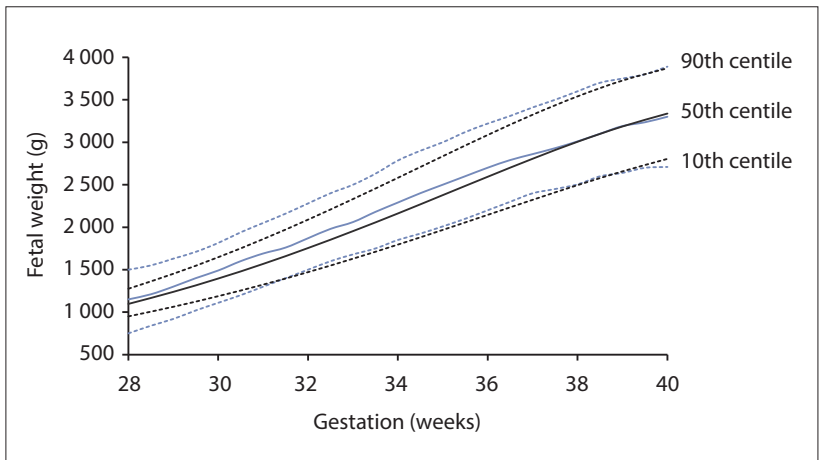

Fig. 1. INTERGROWTH-21st estimated fetal weight (black) compared with Theron-Thompson estimated fetal weight (blue) by gestation.

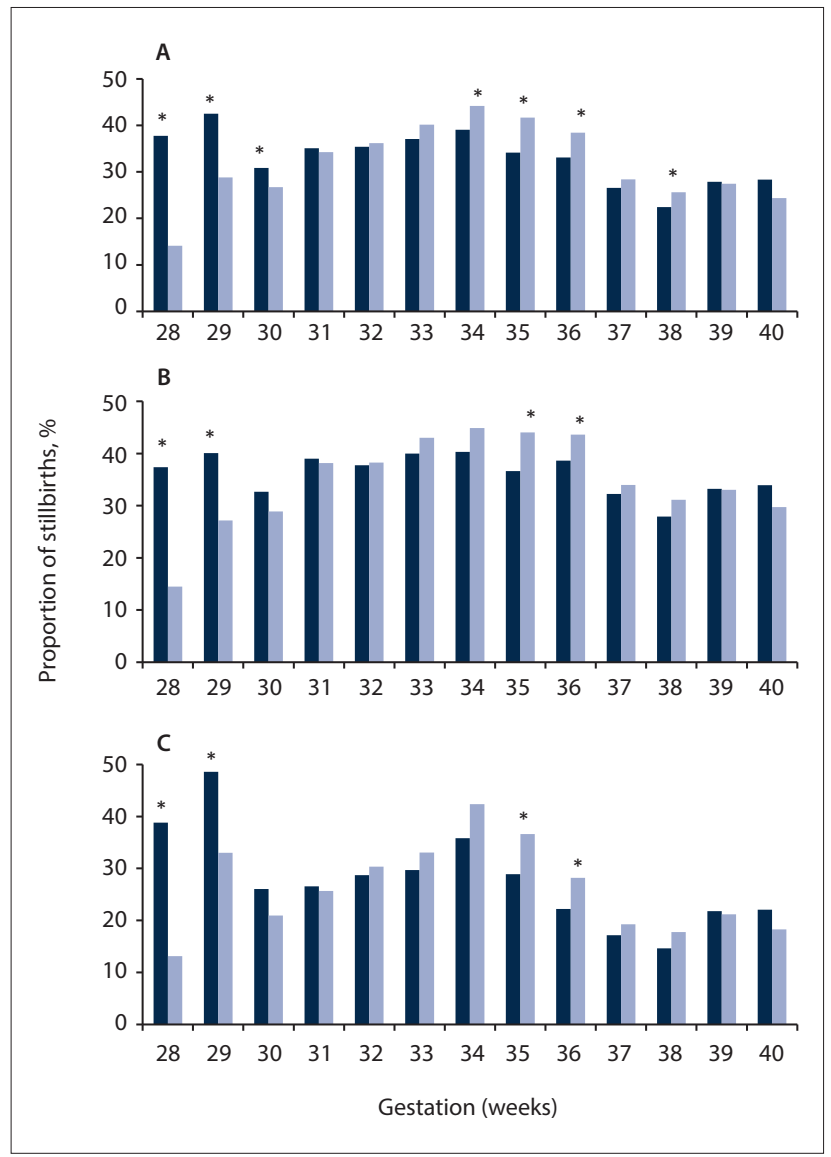

Fig. 2. Proportion of stillbirths that were SGA: (A) all stillbirths ( $\mathrm{N}=14776)$, (B) macerated stillbirths ( $\mathrm{N}=9$ 725), (C) fresh stillbirths ( $\mathrm{N}=5$ 051). ( $S G A=$ small for gestational age; dark blue = INTERGROWTH-21st; light blue = Theron-Thompson; ${ }^{*} \mathrm{p}<0.05$ between INTERGROWTH-21st and TheronThompson.)

\section{Comparison between methods of gestational age determination}

Gestational age was determined by ultrasound in $37.0 \%$ of stillbirths and by clinical examination, the LMP or both in $63.0 \%$. When using Theron-Thompson standards to identify SGA infants, ultrasound classified a larger proportion of stillbirths as SGA (34.0\%) than clinical examination or the LMP $(29.2 \%)(p<0.001)$. The level of agreement for identifying SGA differed by gestational age between INTERGROWTH-21st and Theron-Thompson depending on the method used (ultrasound v. clinical examination/LMP) (Table 3).

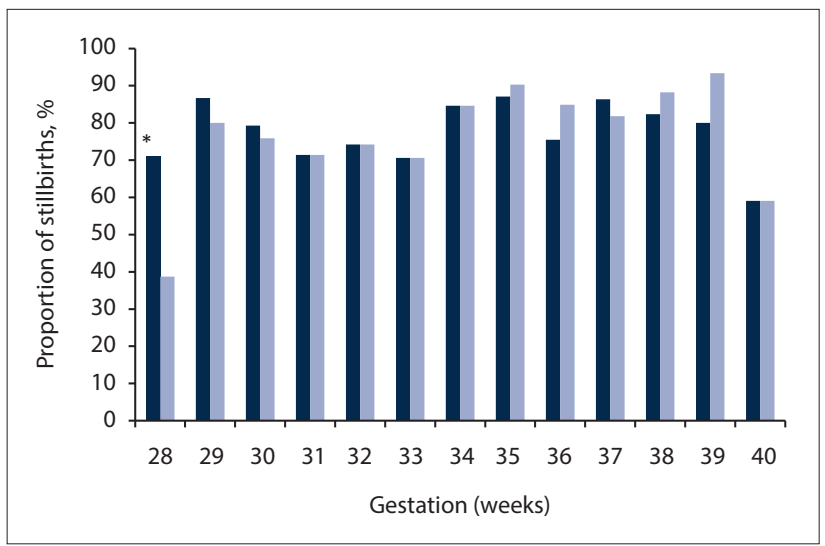

Fig. 3. Proportion of IUGR stillbirths that were SGA $(\mathrm{N}=384)$. (IUGR = interuterine growth restricted; $S G A=$ small for gestational age; dark blue = INTERGROWTH-21st; light blue = Theron-Thompson; ${ }^{*} \mathrm{p}<0.05$ between INTERGROWTH-21st and Theron-Thompson.)

The LMP/clinical examination group had slightly higher levels of agreement between the charts than ultrasound $(99.2 \%$ v. $98.3 \%$, respectively). The lowest levels of agreement between the charts were at 28 weeks (ultrasound $74.8 \%$, clinical examination/LMP 77.2\%).

\section{Discussion}

This large national study found the proportion of stillbirths classified as SGA to be similar between INTERGROWTH-21st and local Theron-Thompson growth charts. Overall across pregnancy, observed agreement and concordance were high. However, when considering gestational age, INTERGROWTH-21st classified more SGA stillbirths at younger gestations and fewer at later gestations compared with Theron-Thompson. A similar trend was observed for IUGR-specific stillbirths. Observed agreement and $\kappa$ varied at each gestational age, with lower agreement at lower gestations and at 34 - 36 weeks.

\section{Proportion of SGA compared with other populations}

One of the aims of INTERGROWTH-21st was that it would enable comparisons in fetal growth and SGA between different settings using a standardised chart. This is the first empirical comparison between the proportion of stillbirths that are SGA in our population and those in other settings using a standardised classification system. The overall proportion of stillbirths that were SGA in our study was similar to that of a UK study (32.6\%). ${ }^{[7]}$ In the UK and NZ, it has been reported that INTERGROWTH-21st underestimated SGA in at-risk infants $s^{[6]}$ and stillbirths ${ }^{[7]}$ compared with local standards. When considering the proportion of SGA stillbirths at a population level, we did not observe any significant under- or overestimation of SGA by INTERGROWTH-21st in the current study.

In the current study, there were no significant differences in the overall proportion of stillbirths classified as SGA between the INTERGROWTH-21st and local methods, and overall observed agreement and $\kappa$ were high. In studies where gestational age is not an important factor and overall SGA prevalence across pregnancy is the outcome of interest, INTERGROWTH-21st may offer a standardised method of comparison between populations, such as in multicountry studies.

\section{SGA by gestational age}

This is the first study to explore the application of INTERGROWTH21 st to local growth curves at each gestational age. INTERGROWTH- 


\begin{tabular}{|c|c|c|c|c|}
\hline \multirow[b]{2}{*}{ Gestation (weeks) } & \multicolumn{2}{|c|}{ Ultrasound } & \multicolumn{2}{|c|}{ Clinical examination/LMP } \\
\hline & Observed agreement, $\%$ & к $(95 \% \mathrm{CI})$ & Observed agreement, \% & $\kappa(95 \% \mathrm{CI})$ \\
\hline Overall (28 - 40) & 98.3 & $0.961(0.954-0.969)$ & 99.2 & $0.982(0.978-0.986)$ \\
\hline 28 & 74.8 & $0.433(0.366-0.499)$ & 77.2 & $0.418(0.357-0.479)$ \\
\hline 29 & 85.6 & $0.696(0.627-0.764)$ & 87.1 & $0.033(0.659-0.788)$ \\
\hline 30 & 94.4 & $0.874(0.829-0.919)$ & 96.7 & $0.916(0.884-0.948)$ \\
\hline 31 & 98.9 & $0.975(0.95-0.999)$ & 99.3 & $0.986(0.969-1.000)$ \\
\hline 32 & 99.0 & $0.978(0.96-0.995)$ & 99.0 & $0.987(0.976-0.997)$ \\
\hline 33 & 95.5 & $0.908(0.867-0.950)$ & 98.1 & $0.958(0.932-0.984)$ \\
\hline 34 & 93.7 & $0.872(0.827-0.917)$ & 95.3 & $0.903(0.873-0.933)$ \\
\hline 35 & 92.9 & $0.850(0.797-0.903)$ & 92.5 & $0.841(0.797-0.885)$ \\
\hline 36 & 94.3 & $0.883(0.842-0.923)$ & 95.4 & $0.895(0.867-0.923)$ \\
\hline 37 & 97.3 & $0.936(0.904-0.969)$ & 98.6 & $0.963(0.945-0.982)$ \\
\hline 38 & 97.0 & $0.929(0.896-0.962)$ & 96.9 & $0.909(0.884-0.934)$ \\
\hline 39 & 99.7 & $0.993(0.98-1.000)$ & 99.5 & $0.987(0.975-1.000)$ \\
\hline 40 & 95.8 & $0.904(0.851-0.957)$ & 96.3 & $0.899(0.861-0.938)$ \\
\hline
\end{tabular}

21st classified more SGA stillbirths at earlier gestations and fewer at later gestations. Observed agreement and concordance were also lower at these gestations. The largest discrepancies for 10th-centile cut-points between the two charts were at the younger gestations. At these gestations, INTERGROWTH-21st identified a much larger proportion of SGA stillbirths than Theron-Thompson. Previous studies have not considered the impact of gestational age on observed agreement for different methods of SGA identification. In the current study, this observation would have been masked if comparisons were only made overall across pregnancy, indicating the importance of assessment by specific gestational age.

The reasons for the differences between classification by INTERGROWTH-21st and Theron-Thompson at different gestations are unclear, but ethnicity, population-specific growth differences and inaccuracies in gestational age/weight may all play a role.

Differences in the composition of ethnicities in the samples used to develop Theron-Thompson and INTERGROWTH-21st may contribute to the differences seen in the fetal growth charts in identifying SGA. INTERGROWTH-21st was based on women from eight countries aged 15 - 35 years, with no diagnosed morbidity, who were well nourished, lived in urban areas and did not smoke. ${ }^{[1]}$ The premise was that these women were 'low-risk' healthy women who represented 'normal physiological growth' and comprised a multiethnic sample. ${ }^{[11]} \mathrm{SA}$ also has a multiethnic population with more than 10 ethnic groups, none of which were included in the development of INTERGROWTH-21st. Several studies have observed ethnic differences in the application of INTERGROWTH21st. An NZ study found that compared with INTERGROWTH-21st, customised criteria identified $2-3$ times as many SGA infants in Maori, Pacific, European and Asian pregnancies. ${ }^{[6]}$ The National Institute of Child Health and Human Development fetal growth studies found significant differences in fetal growth by race/ethnicity in US women and has since developed race-specific charts. ${ }^{[12]}$ It is possible that racial/ethnic differences in fetal growth across pregnancy are present and may account for the differences seen at specific gestational ages.

The differences observed may also extend to a population level. There are recognised differences in optimal perinatal outcome achieved at different birth weights in different populations. ${ }^{[13,14]}$
Different populations have different birth-weight distributions and optimal fetal growth standards as well as perinatal mortality curves. $^{[13,14]}$ The mortality curves are shifted in the same direction as birth weight. ${ }^{[13]}$ The findings of the current study also support the notion that differences in fetal growth patterns resulting in adverse outcome (stillbirth) are present when using population-specific growth charts as opposed to internationally derived charts.

A possible alternative explanation for differences across gestational age is that there are greater inaccuracies in the determination of gestational age and weight at younger gestations, leading to higher levels of disagreement between INTERGROWTH-21st and Theron-Thompson (larger differences at smaller gestations). Using ultrasound to determine intrauterine estimates of fetal weight is known to be less accurate at $<2000 \mathrm{~g},{ }^{[15]}$ and symphysis-fundal height and the LMP have known inherent issues in determining gestational age and size. ${ }^{[16]}$ We sought to reduce these issues by including only cases in which gestational age determination was 'certain'. One would expect that symphysis-fundal height/LMP would have a greater number of inaccuracies in determining gestational age and more difficulty in determining fetal weight given small fetal size. However, this was not reflected in our data, with greater agreement between the charts for gestational age determined by LMP/clinical examination at most gestations. We also had a large sample size (>1 900 stillbirths) at gestations of 28 - 29 weeks, therefore not compromising the power. Overall it is unlikely that the differences seen at earlier gestations are an artefact of inaccuracies in gestational age or weight determination at younger gestations.

\section{Public health implications}

If INTERGROWTH-21st charts were to be adopted clinically in SA as a method to identify 'high-risk' women based on fetal growth, this would have implications for maternal health service systems. Women in SA receive antenatal care at the community level with a nurse/midwife and are up-referred to obstetric specialist care at the district level if identified as high risk. ${ }^{[17]}$ One of the criteria for high risk is SGA and/or slowing growth velocity as classified through growth charts. Specialised obstetric care and serial ultrasound are only available in high-risk pregnancies. The adoption of INTERGROWTH-21st would increase the number 
of women classified as high risk and up-referred for obstetric antenatal at lower gestations ( 28 - 30 weeks), increasing demand on already under-resourced obstetric services. Increased medicalisation without prevention of adverse perinatal outcomes due to suboptimal diagnostic accuracy of antenatal growth charts has been highlighted previously. ${ }^{[3]}$ It is unclear whether identifying more pregnancies as SGA at earlier gestations would result in a decrease in stillbirths or increased medicalisation without prevention of adverse outcomes.

Conversely, based on our findings, the application of INTERGROWTH-21st classification to the SA population would decrease the proportion of women classified as high risk due to SGA at gestations of 34 - 36 weeks. This gestational period has been identified as a crucial period for stillbirths in $\mathrm{SA},{ }^{[9]}$ and has also been identified as a high-risk period for women who do not receive antenatal care during this time. ${ }^{[18]}$ It is possible that not recognising these pregnancies as SGA and therefore continuing to consider them as low risk would result in an increased stillbirth rate during this period. Further, the timing of ultrasound assessment (only available to high-risk women in SA) for SGA in late pregnancies becomes important owing to the slowing of growth velocity of some fetuses near term, thus meeting the criteria for SGA for the first time in late pregnancy. ${ }^{[19]}$ An increased number of these fetuses may be missed if a less sensitive INTERGROWTH-21st growth chart is used at this time point.

From a public health perspective, an optimal fetal growth standard is one that most accurately identifies fetuses at risk of poor perinatal outcome. ${ }^{[13]}$ Population-specific growth standards and customised growth charts are arguably more appropriate than generalised growth standards owing to their ability to take into account the fact that optimal perinatal outcomes are achieved at different birth weights for different populations. The current study observed differences in the population-specific charts in recognising SGA fetuses where adverse outcomes occurred (all stillbirths and IUGR stillbirths) compared with international standards at different gestational ages. The impact of the differences by gestational age on adverse perinatal outcomes would need to be considered if SA were to adopt INTERGROWTH21 st in place of local standards as a method of classifying SGA pregnancies. Currently, customised growth charts based on an SA population are not available.

\section{Study strengths and limitations}

While it is important to identify SGA fetuses, it must also be recognised that growth charts only identify pregnancies requiring further investigation. A fetus that is classified as SGA may not necessarily be pathologically growth restricted, and may be healthy with normal growth that just happens to fall in the lowest 10th centile on growth charts. ${ }^{[20]}$ In addition, it must be acknowledged that growth restriction may also occur in AGA pregnancies. ${ }^{[2]}$ This was observed in our study, where one-fifth of stillbirths with IUGR as the primary cause of death were AGA (using either growth chart). Umbilical artery Doppler measurements using an inexpensive hand-held device such as the Umbiflow can be used to identify such cases. ${ }^{[21]}$

This study focused on stillbirths only, representing pathological pregnancies. It is likely that SGA estimates for macerated stillbirths are overestimated using all criteria, as the death may have occurred up to 3 weeks earlier ${ }^{[22]}$ and the fetus may therefore have been AGA at the time of death. Fresh stillbirths are likely to represent more accurate estimates for SGA, as the time difference between fetal death and delivery is not considerable. Future studies should consider the prevalence of SGA in live births to reduce the overestimation of SGA. We were also only able to examine pregnancies at $28-40$ weeks, as Theron-Thompson growth charts are not reliable for gestations
$<28$ weeks $^{[10]}$ and INTERGROWTH-21st estimated fetal weight is only available up to 40 weeks.

This was one of the largest studies in low- to middle-income countries (LMICs) examining the proportion of SGA stillbirths using INTERGROWTH-21st standards. It is unclear how these results can be generalised to other LMICs owing to differences in ethnicity and fetal growth at a population level, which may influence the classification of SGA in stillbirths. ${ }^{[6,12]}$ The study has highlighted the need for each country to carefully examine and consider the application of INTERGROWTH-21st in its own context, specifically at each gestational age, before adopting these standards for clinical use or use in epidemiology.

\section{Conclusions}

Our study showed differences in the estimated proportion of stillbirths considered SGA at each gestational age, depending on the growth chart used, that have not been considered in previous studies. This finding highlights the importance of future studies considering SGA at each gestational age and not simply comparing proportions across the entire pregnancy period. The development of an international standard is essential to compare the prevalence of SGA between countries. The results of the current study have public health implications for identifying infants at risk of stillbirth antenatally in a low-resource setting. Each country must carefully consider the impact of using INTERGROWTH-21st owing to the issues raised in this study pertaining to gestational age and potential ethnic and population differences in optimal fetal growth.

\section{Declaration. None}

\section{Acknowledgements. None.}

Author contributions. TL: conceptualised study, analysed data, drafted manuscript, edited manuscript. LN: analysed data/supervised data analysis, revised/edited manuscript. DP: supervised data analysis, revised/ edited manuscript. GT: revised/edited manuscript. RCP: revised/edited manuscript.

Funding. TL is supported by an Australian Postgraduate Award and University of Western Australia top-up scholarship. LN is funded by a National Health and Medical Research Council of Australia Early Career Fellowship. This work was funded by a University of Western Australia Research Collaboration Award.

Conflicts of interest. None.

1. Lindqvist PG, Molin J. Does antenatal identification of small-for-gestational age fetuses significantly improve their outcome? Ultrasound Obstet Gynecol 2005;25(3):258-264. https://doi.org/10.1002/ uog. 1806

2. Poljak B, Agarwal U, Jackson R, Alfirevic Z, Sharp A. Diagnostic accuracy of individual antenatal tools for prediction of small-for-gestational age at birth. Ultrasound Obstet Gynecol 2017;49(4):493-499. https://doi.org/10.1002/uog.17211

3. Papageorghiou AT, Ohuma EO, Altman DG, et al. International standards for fetal growth based on serial ultrasound measurements: The Fetal Growth Longitudinal Study of the INTERGROWTH-21st Project. Lancet 2014;384(9946):869-879. https://doi.org/10.1016/S0140-6736(14)61490-2

4. Villar J, Knight HE, de Onis M, et al. Conceptual issues related to the construction of prescriptive standards for the evaluation of postnatal growth of preterm infants. Arch Dis Child 2010;95(12):1034standards for the evaluation of postnatal growt

5. McCarthy EA, Walker SP. International fetal growth standards: One size fits all. Lancet 2014;384(9946):835-836. https://doi.org/10.1016/S0140-6736(14)61416-1

6. Anderson NH, Sadler LC, McKinlay CID, McCowan LME. INTERGROWTH-21st vs customized 6. Anderson NH, Sadler LC, McKinlay CJD, McCowan LME. INTERGROWTH-21st vs customized
birthweight standards for identification of perinatal mortality and morbidity. Am J Obstet Gynecol 2016;214(4):509.e1-509.e7. https://doi.org/10.1016/j.ajog.2015.10.931

Poon LC, Tan MY, Yerlikaya G, Syngelaki A, Nicolaides KH. Birth weight in live births and stillbirths. Ultrasound Obstet Gynecol 2016;48(5):602-606. https://doi.org/10.1002/uog.17287

8. Cheng YKY, Leung TY, Lao TTH, Chan YM, Sahota DS. Impact of replacing Chinese ethnicity-specific fetal biometry charts with the INTERGROWTH-21st standard. BJOG 2016;123(S3):48-55. https://doi. org/10.1111/1471-0528.14008

9. Lavin T, Preen DB, Pattinson R. Timing and cause of perinatal mortality for small-for-gestationalage babies in South Africa: Critical periods and challenges with detection. Matern Health Neonatol Perinatol 2016;2:11-21. https://doi.org/10.1186/s40748-016-0039-4

10. Theron GB, Thompson ML. A centile chart for birth weight for an urban population of the Western Cape. S Afr Med J 1995;85(12):1289-1292. 
11. Villar J, Ismail LC, Victora CG, et al. International standards for newborn weight, length, and head circumference by gestational age and sex: The Newborn Cross-Sectional Study of the head circumference by gestational age and sex: The Newborn Cross-Sectional Study of the
INTERGROWTH-21st Project. Lancet 2014;384(9946):857-868. https://doi.org/10.1016/S0140INTERGROWT(14)60932-6

12. Grantz KL, Hediger ML, Liu D, Buck Louis GM. Fetal growth standards: The NICHD fetal growth study approach in context with INTERGROWTH-21st and the World Health Organization Multicentre Growth Reference Study. Am J Obstet Gynecol 2018;218(2S):S641-S655.e28. https://doi. org/10.1016/.j.jog.2017.11.593

13. Reeves S, Bernstein IM. Optimal growth modeling. Semin Perinatol 2008;32(3):148-53. https://doi. org/10.1053/.semperi.2007.11.001

14. Graafmans WC, Richardus JH, Borsboom GJ, et al. Birth weight and perinatal mortality: A comparison of 'optimal' birth weight in seven Western European countries. Epidemiology 2002;13(5):569-574

15. Barel O, Vaknin Z, Tovbin J, Herman A, Maymon R. Assessment of the accuracy of multiple sonographic fetal weight estimation formulas: A 10-year experience from a single center. J Ultrasound Med 2013;32(5):815-823. https://doi.org/10.7863/jum.2013.32.5.815

16. Peter JR, Ho JJ, Valliapan J, Sivasangari S. Symphysial fundal height (SFH) measurement in pregnancy for detecting abnormal fetal growth. Cochrane Database Syst Rev 2015, Issue 9. Art. No.: CD008136. https://doi.org/10.1002/14651858.CD008136.pub3

17. National Department of Health, South Africa. Guidelines for Maternity Care in South Africa: A Manual for Clinics, Community Health Centres and District Hospitals. 4th ed. Pretoria: Government Printer, 2015
18. Lavin T, Pattinson RC. Does antenatal care timing influence stillbirth risk in the third trimester? A secondary analysis of perinatal death audit data in South Africa. BJOG 2018;125(2):140-147. https:// doi.org/10.1111/1471-0528.14645

19. Sovio U, White IR, Dacey A, Pasupathy D, Smith GCS. Screening for fetal growth restriction with universal third trimester ultrasonography in nulliparous women in the Pregnancy Outcome Prediction (POP) study: A prospective cohort study. Lancet 2015;386(10008):2089-2097. https://doi.org/10.1016 S0140-6736(15)00131-2

20. Gardosi J, Mul T, Mongelli M, Fagan D. Analysis of birthweight and gestational age in antepartum stillbirths. Br J Obstet Gynaecol 1998;105(5):524-530.

21. Nkosi S, Makin J, Vilikazi B, Hlongwane T, Pattinson R. Screening and managing low risk pregnant population using continuous wave Doppler ultrasound in a low income population. S Afr Med J 2019;109(5):347-352. https://doi.org/10.7196/SAMJ.2019.v109i5.13611

22. Genest DR, Singer DB. Estimating the time of death in stillborn fetuses: III. External fetal examination: A study of 86 stillborns. Obstet Gynecol 1992;80(4):593-600. 